\title{
Late stages of stellar evolution in population models.
}

\section{Claudia Maraston}

Institute of Cosmology and Gravitation, University of Portsmouth, Dennis Sciama Building, Burnaby Road, Portsmouth PO1 3FX. email: claudia.maraston@port.ac.uk

\begin{abstract}
My contribution to Roger's celebration symposium focuses on the treatment of late stellar evolutionary phases in stellar population models, reviewing the state of art and discussing some very recent developments, ranging from local stellar clusters up to distant galaxies at high redshift. I shall focus in particular on the Thermally Pulsating Asymptotic Giant Branch, about which a vivid discussion has been ongoing since a few years. I shall present renewed evidence in favour of a sizable contribution from this phase for matching the observed spectral energy distribution of distant massive galaxies. I shall also discuss the possible reasons why such a conclusion has been controversial in the recent literature. Stellar population models are the magic tool to shape the physics of galaxies out of their observed light, and enter virtually all papers presented at this symposium. In a collective effort to properly treat all relevant aspects of the modelling, we split the discussion into six contributions given by experts in the field, as our present to Roger and his outstanding career.
\end{abstract}

Keywords. stars: evolution - galaxies: evolution

\section{Introduction}

Late stages of stellar evolution refer to the period of (relatively) rapid burning of $\mathrm{Hy}$ drogen and/or Helium in shells outside the core, following the exhaustion of the main stellar fuel, the central Hydrogen burning. The occurrence and duration of such phases depends on the initial stellar mass. High-mass stars $\left(M>8 M_{\odot}\right)$ go rapidly into central He-burning and after a short phase of Red or Blue supergiants, end as SN-II. In intermediate-mass stars $\left(2<M<8 M_{\odot}\right)$ the most relevant late evolutionary phase is the Asytmptotic Giant Branch (AGB), while low-mass stars $\left(M<2 M_{\odot}\right)$ mark the start of prolongued Red Giant Branch (RGB) and Horizontal Branch (HB). Fig. 1 gives a snapshot of late evolutionary phases on the HR diagram of an old population. $\dagger$

Late evolutionary phases are featured by short $(t<1 \mathrm{Gyr})$ lifetimes, a high luminosity, temperatures ranging to extreme values (the tip of the RGB and the AGB extend to very cold temperatures, $T_{\text {eff }}<4000 K$ and hot $\mathrm{HB}$ runs to hot values of temperatures, $\left.T_{\text {eff }}>>10000 \mathrm{~K}\right)$. The extreme regimes coupled with mass-loss, rotation and envelope instabilities make the theoretical modelling complicated. Mass-loss in particular hampers a clean prediction of the full evolution over key late phases such as the pulsating major part of the AGB, so-called Thermally-Pulsating Asymptotic Giant Branch (TP-AGB), and the Horizontal Branch. In order to encompass these difficulties and help calculating models which could be applied to galaxies with some confidence, Renzini \& Fusi-Pecci (1988) suggested to calibrate the unknown of stellar evolution on the simplest stellar systems known in order to obtain empirical estimates of a few relevant parameters, such as the efficiency of mass-loss, the fuel burnt, etc. which could then be used to

$\dagger$ There are other late phases, e.g. the UV-upturn, which are not treated here for reasons of space. 


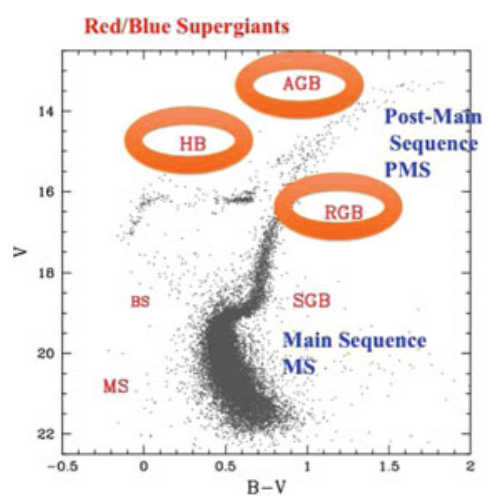

Figure 1. Displacement of late stellar evolutionary phases, RGB, HB, AGB (circled in orange) and red/blue supergiants, labelled on the upper axis, onto the HR diagram of an old population. Adapted from Maraston (2005).

constrain theoretical stellar models. A similar approach was then extended to stellar population (i.e. integrated) models by using whole star clusters as proto-typical single burst (SSP) populations (for the TP-AGB Maraston 1998; Maraston \& Thomas 2000, for the HB morphology). Even if nowadays the actual nature of most star clusters as single generations is questioned as often multiple Main Sequences are detected in Milky Way and Magellanic Clouds objects (e.g. Piotto et al. 2007), star clusters are still simpler than galaxies.

They do have a disadvantage though, which is their relatively low number of stars $\left(\sim 10^{4 \div 6}\right)$ which makes the sampling of the short late stelar stages hard against stochastical magnitude fluctuations. Massive galaxies hosting nearly coeval stellar populations are immune from fluctuations. This fact was the pillar for using passive galaxies at high redshift as a showcase for the testing of various assumptions regarding the duration and fuel consumption of the TP-AGB (Maraston et al. 2006), the shortest $\left(t \sim 10^{6} \mathrm{yr}\right.$ ) and most luminous late phase of stellar evolution.

The energetic and lifetime we include in population models directly impact on the physical properties that are derived or predicted for galaxies, hence on our understanding of galaxy evolution. In the following I shall briefly review the most important facts of each late stellar phases, allowing more space to the highly debated TP-AGB and new results first presented at this symposium. I shall then provide a brief summary.

\section{Massive stars: Red or Blue supergiants?}

The fate of a massive star as a red or blue supergiant depends on assumptions for convective overshooting, rotation and mass-loss (see e.g. Chiosi \& Maeder 1986). Various stellar models include this late stage of massive stars with a different typical temperature, which then reflects itself in a different integrated spectrum of stellar populations. Fig. 2 (left-hand panel) compares models by Maraston (2005, hereafter M05) (red) and Bruzual \& Charlot (2003, hereafter BC03) (blue) which are based on Geneva and Padova stellar tracks, respectively, at ages of 1 and 10 Myr. The effect of the temperature of the supergiant phase is evident at 10 Myr models, where the M05 model has more nearIR flux than the $\mathrm{BC} 03$ one, because the phase is spent at cooler temperatures. How to disentangle the correct temperature? The right-hand panel is taken from Marigo et al. (2008), which uses the $V-K$ integrated colour vs age as a diagnostic to compare stellar population models (lines) with Magellanic Clouds star clusters (symbols). The youngest 

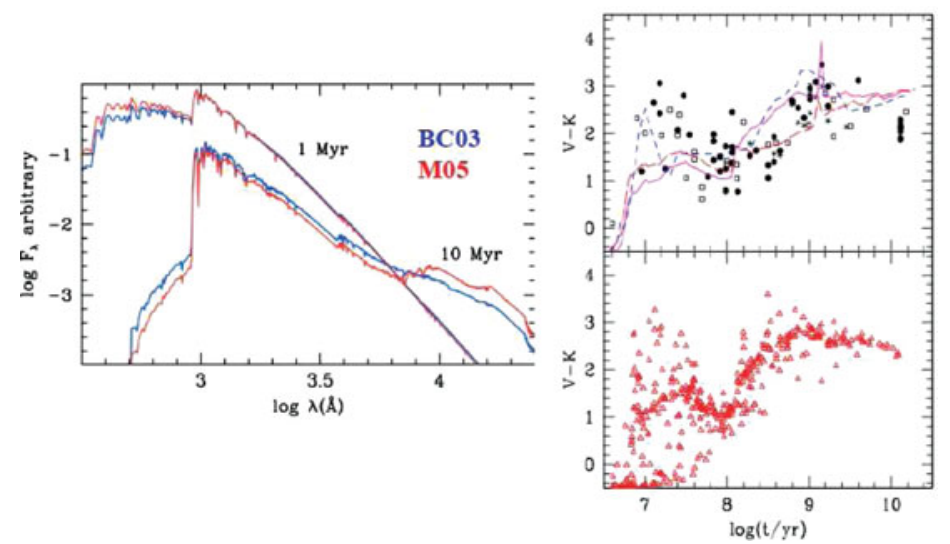

Figure 2. The left-hand panel shows young SSP models by M05 (red) and BC03 (blue), which are based on different stellar evolutionary tracks. The right-hand panel from Marigo et al. (2008) shows how SSP models compare to the $V-K$ colours of Magellanic Clouds GCs. M05 are the blue lines.

clusters reach very red colours, which are better consistent with a red supergiant phase as in the M05 models (blue lines). Marigo et al. (2008) also shows in the lower panel the effect of stochastical fluctuations on their stellar population models based on Padova tracks.

\section{The temperature of the Red Giant Branch.}

Maraston (2005, Figure 9) shows how different stellar models predict different typical temperatures for the RGB, the tracks by the Padova database being the coolest and those from the Teramo's one the warmest. Fig. 27 in the same paper shows the RGB temperature effect on the integrated spectrum of a stellar population model. The coolest tracks make the integrated SED redder which, in terms of galaxy properties, implies the derivation of a lower total metallicity or a younger age. The RGB flux vs $T_{\text {eff }}$ also affects the derivation of the stellar Initial Mass Function (IMF) from near-IR indices (see C. Conroy; R. Smith, this volume). The RGB track depends on several input physics, notably on the mixing-length which is one of the parameters to be calibrated with observations (see discussion in M05). Interesting plots are shown in McDonald et al. (2011) who use various stellar models to derive the properties of stars in the metal-rich MW GC 47 Tuc (Fig. 3). It can be seen that Padova tracks are indeed too cold at the nominal cluster metallicity (left-hand panel), whereas those by the Darmouth $\dagger$ database fit better (righthand panel). However, the same tracks including an $\alpha / F e$ ratio as measured in 47 Tuc (lower right-hand panel) perform slightly worse than the solar-scaled ones! Progress is needed to correctly predict the effect of non-solar chemical mixtures on stellar evolution and model atmospheres (see D. Thomas and R. Schiavon, this volume).

\section{The morphology of the Horizontal Branch.}

It is well known that the late evolutionary phase of HB manifests itself in a variety of 'morphologies', thought to be mostly (but not only) related to metallicity (see references in Maraston \& Thomas (2000)). Stellar population models calculated for various HB

$\dagger$ Due to space constraints we cannot provide full citations and refer the reader to the original papers. 

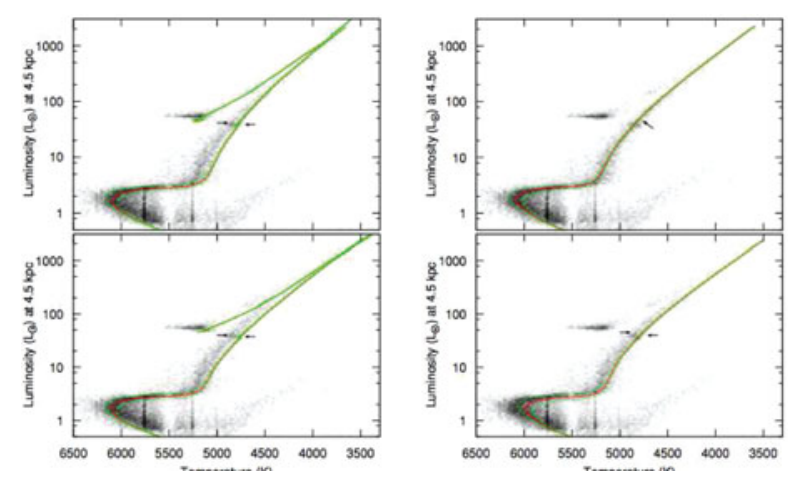

Figure 3. Comparison of theoretical isochrones, from Padova and Darmouth (left-hand and right-hand panels, respectively) to the observed CMD of the metal-rich GC 47 Tuc. From McDonald et al. (2011).

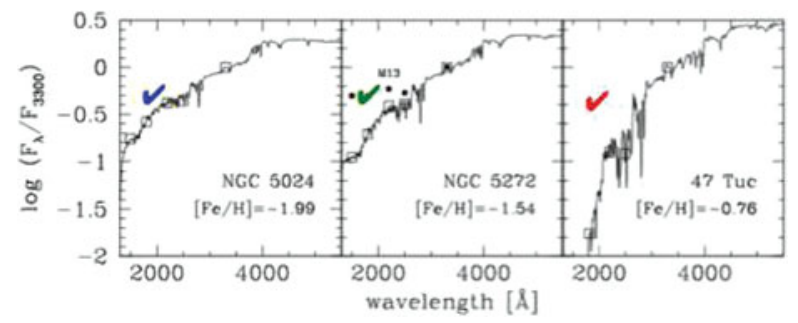

Figure 4. Theoretical SED of stellar population models with the same age (14 Gyr) and various $[\mathrm{Z} / \mathrm{H}]$ matching the variety of $\mathrm{HB}$ morphologies observed in MW GCs, blue, intermediate and red from left to right. From Maraston \& Thomas (2000).

morphologies (as in M05) have a larger degree of flexibility to experiment with observations. In the M05 models, the tunable parameter is the mass-loss along the RGB, which allows to obtain hotter tracks without varying the evolutionary mass (i.e. the age) of the model. Fig. 4 plots data for three MW GCs having different metallicities, a range of HB morphologies, from blue to red (from left to right) and a similar age. Stellar population models are best-fitted to them, in which the HB morphology has been adapted to the observed case, by playing with the mass-loss efficiency.

\section{The infamous TP-AGB phase and high-redshift galaxies.}

The TP-AGB phase and its effects on stellar population models and galaxies has gained the honour of the press several times since the works of Maraston (1998, 2005); Maraston et al. (2006). Several reviews are available (e.g. Maraston 2013), the content of which will not be repeated here. Briefly, Maraston $(1998,2005)$ used Magellanic Clouds GCs to calibrate the energetic of the TP-AGB and use the observed colours of the same clusters to fix the flux of model SEDs. ? (and others) find the M05 models to match well the full SEDs of galaxies at high- $z$, in particular those in mostly passive evolution. Recently, Conroy \& Gunn (2010) have questioned the above mentioned calibration, based on newer MC GCs data and especially on a new age scale for the observed clusters, concluding that M05 models are too red, in other words contain too much TP-AGB. On a similar path, Kriek et al. (2010) concluded that BC03 models fit observed galaxy SEDs much better than M05 models and indicate in the high TP-AGB of the M05 models the cause of the misfit. 

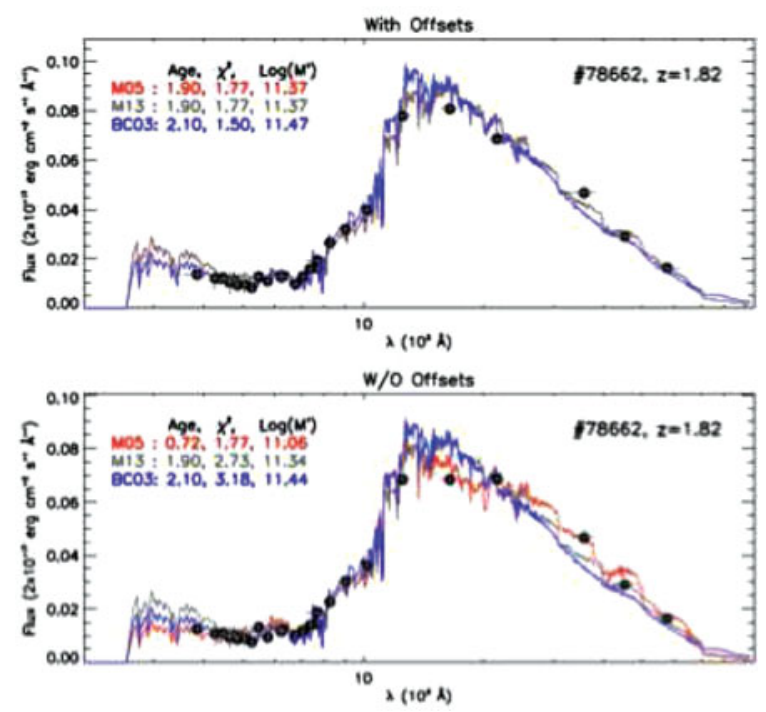

Figure 5. Effect of model-dependent photometric offsets on SED fits. Upper panel: data with model-dependant offsets favour BC03 models (blue line). Bottom panel: the removal of the offsets changes the observed SED and favour M05 (red line). Adapted from Capozzi et al. (2015).

The Kriek et al. (2010) sample contain $\sim 66$ galaxies with photometric redshift, preselected and stack as to match a post-starburst criterium defined via rest-frame colours. Their approach is quite dissimilar to that of Maraston et al. (2006), hence Capozzi et al. (2015) re-examine our earlier results, who strictly used galaxies with spectroscopic redshifts only, by re-analysing the same objects with new photometry available in the meantime. Moreover, the sample of spectroscopic galaxies was augmented with $\sim 50$ more objects from the COSMOS survey pre-selected to be in nearly passive evolution as those of Maraston et al. (2006). It should be noticed that this selection is applied initially, but then the data are fit with models calculated with a large variety of star formation histories and reddening, i.e. the galaxies are left free to move away from this initial pre-selection, wish they wanted to.

Capozzi et al. (2015) fully confirm the results of Maraston et al. (2006), namely that the strong TP-AGB M05 models fit the data better than BC03 models, and the inclusion of reddening as an additional free parameter makes the fits with both models comparable. These results are ad odd with Kriek et al. (2010). Capozzi et al. (2015) discuss another interesting effect. It is found that often raw photometric data are applied offsets to improve the performance of photometric redshifts. These offsets are calculated using stellar population models. It should then go without saying that 'model-shaped' data should not be further used to perform comparisons with models, as obviously the models used to obtain the offsets would appear favoured. The case is shown in Fig. 5 and 6 . It remains to be assessed whether the use of BC03-based off-setted photometry is in part responsible for the discrepant results just discussed.

Finally, the new version of M05-type models with reduced TP-AGB fuel as re-calibrated after Conroy \& Gunn (2010) (shown in Noell et al. 2013) are not found to match the high- $z$ galaxies better than M05. 

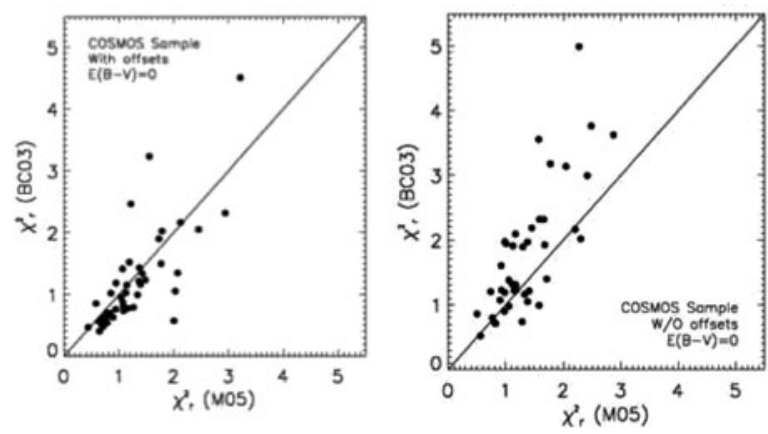

Figure 6. The effect of model-dependent photometric offsets to galaxy data on the goodness of SED fits of models to the same data. Model-dependant offsets favour the models on which the offsets were calculated (BC03, left-hand panel). The removal of the offset changes the trend and now M05 is favoured. Adapted from Capozzi et al. (2015).

\section{Outlook}

A proper description of late evolutionary stages in stellar population models requires the calibration of free parameters with data, and stellar clusters have been regarded as the ideal objects in this game. While the procedure still seems to hold for most phases, an interesting discrepancy emerges between star clusters and massive galaxies in case of the TP-AGB phase, where galaxies require a large effect from this stellar phases.

\section{Acknowledgements}

Thanks to Michele and Stephane for organising an outstanding conference, and to Roger for being such a brilliant scientist and a great friend.

\section{References}

Bruzual, G. \& Charlot, S. 2003, MNRAS, 344, 1000

Capozzi, D. et al., 2015, MNRAS, submitted

Chiosi, C. \& Maeder, A. 1986, ARA\&A, 24, 329

Conroy, C. \& Gunn, J. 2010, ApJ, 712, 883

Kriek, M., et al. 2010, ApJ, 722, L64

Maraston, C., 1998, MNRAS, 300, 872

Maraston, C., 2005, MNRAS, 362, 799

Maraston, C., 2013, in "The Intriguing Life of Massive Galaxies, Proceedings of the International Astronomical Union, IAU Symposium, Volume 295, pp. 272-281

Marigo, P., et al. 2008, å, 482, 883

Maraston, C., Thomas, D. 2000, ApJ, 541, 126

Maraston, C., Daddi, E., \& Renzini, A. 2006, ApJ, 652, 85

McDonald, A., et al. 2011, ApJS, 193, 23

Noel, N., et al. 2013, ApJ, 772, 58

Piotto, G., et al. 2007, ApJ, 661, L53

Renzini, A. \& Fusi-Pecci, F. 1988, ARA\& A, 26, 199 